\title{
First record to Brazil of one genus and seven species of Psychodidae (Diptera) with further new records for 10 countries on the Neotropics
}

\author{
Danilo Pacheco Cordeiro ${ }^{1,2,3}$ \\ 1 Instituto Nacional da Mata Atlântica (INMA), Museu de Biologia Professor Mello Leitão (MBML). Santa Teresa, ES, Brasil. \\ ${ }^{2}$ Instituto Nacional de Pesquisas da Amazônia (INPA), Coordenação de Biodiversidade (CBI0). Manaus, AM, Brasil. \\ ${ }^{3}$ ORCID: http://orcid.org/0000-0002-3085-8628.E-mail:d.pacheco.c@gmail.com
}

\begin{abstract}
With a big territory and variety of biomes, Brazil is one of the most diverse countries in the World, with insects massively contributing to this diversity. Although presenting impressive numbers, many groups are poorly known concerning their diversity and distribution. Also, the knowledge of the species diversity is very heterogeneous when comparing Brazilian states and regions. With a recent review of part of Lawrence Quate's collection deposited at Natural History Museum of Los Angeles County, more than 70 new geographical records for Psychodidae species were found on the Neotropics, including one genus (Eurygarka) and seven species first recorded to Brazil. The complete known distribution of these species was compiled and is presented along with new records for other 11 countries of the Neotropical region. On the Brazilian territory, most of the new records are for the state of Rondônia, on the northern region, followed by Minas Gerais, on the southeastern region.
\end{abstract}

Key-Words. Moth flies; Neotropical Region; Psychodinae; Brazilian fauna.

\section{INTRODUCTION}

Considering insect diversity, Brazil has already been suggested as the most diverse country in the World, for its big territory and variety of biomes (Rafael et al., 2012). Diptera is the third most diverse order of Brazilian fauna, only behind Coleoptera and Lepidoptera. The current knowledge of the Brazilian Diptera fauna accounts 11,217 valid species in 1,950 genera and 99 families (Rafael, 2019; accessed 10/10/2019).

Psychodidae (moth flies and sand flies) is the fifth family in number of known species in Brazil, with 546 valid names, behind Phoridae (851), Tachinidae (777), Chironomidae (631) and Syrphidae (588) (Rafael, 2019; accessed 10/10/2019). Among the five subfamilies occurring in Brazil, Phlebotominae is the largest subfamily in number of known species, with more than 275 valid names. This subfamily has historically received more attention due to its medical importance: females are hematophagous, and many species are potential vectors of etiological agents of Leishmaniasis, arboviruses and Bartonellosis (Rangel \& Lainson, 2009). Psychodinae is the second in number of known species in Brazil, and has the most varied biology, with larvae occupying a multitude of habitats (Wagner \& Ibáñez-Bernal, 2009).
The geographic distribution of American sand flies has been being investigated by many studies, especially in the last three decades, with detailed distribution of sand flies in Brazilian territory (Aguiar \& Vieira, 2018). On the opposite side the distribution of species of other subfamilies is still poorly known, and the knowledge of the diversity of Psychodidae (except of Phlebotominae) in Brazil is very heterogeneous when comparing states and regions (Bravo \& Araújo, 2014). Knowing the diversity and distribution of species is fundamental to help studies on biogeography and conservation.

A recent visit to Los Angeles County Museum to collect information of Brazilian Psychodidae specimens deposited in their collection, allowed the study of the collection left by Lawrence Quate, which has many well-preserved Neotropical material. Herein, more than 70 new distributional records are made for Psychodidae species in the Neotropics, including one genus and seven species first recorded to Brazil.

\section{MATERIAL AND METHODS}

All specimens are slide mounted in Canada balsam and deposited at LACM Entomological collection. Images were taken on a Keyence VHX-5000 digital microscope. 
Table 1. Species with new records for countries territory and their known distribution in the Neotropics. Abbreviations of Countries follows International Organization for Standardization 2-letter codes. $\mathbf{N R}=$ new record, $\mathrm{PR}=$ previous record, $\mathrm{TL}=$ type locality, ${ }^{*}$ type locality outside the Neotropics.

\begin{tabular}{|c|c|c|c|c|c|c|c|c|c|c|c|c|c|}
\hline & GT & NI & CR & PA & BS & PR & CO & VE & SR & GF & EC & PE & BR \\
\hline Alepia ancylis & & & & & & & & NR & & & NR & $\mathrm{TL}$ & NR \\
\hline Caenobrunettia sarculosa & & & PR & $\mathrm{TL}$ & & & NR & & PR & & & PR & PR \\
\hline Platyplastinx culmosus & & & & & & & & & & & $\mathrm{TL}$ & & NR \\
\hline Psychoda buxoides & NR & & $\mathrm{TL}$ & PR & & & & NR & NR & & & NR & PR \\
\hline Psychoda entolopha & NR & NR & $\mathrm{TL}$ & & & & & NR & & & & NR & NR \\
\hline Psychoda flagellata & & PR & $\mathrm{TL}$ & PR & & & & & NR & NR & & & NR \\
\hline Psychoda laticaula & & PR & $\mathrm{TL}$ & & & & & NR & & & & NR & PR \\
\hline Psychoda litotes & & PR & $\mathrm{TL}$ & & & & & NR & & NR & & & $P R$ \\
\hline Psychoda obeliske & NR & PR & $\mathrm{TL}$ & & & NR & & NR & NR & NR & & NR & NR \\
\hline Psychoda savaiiensis* & NR & PR & PR & PR & NR & PR & & NR & NR & NR & & NR & PR \\
\hline Psychoda setigera* & & PR & PR & & & & & NR & & NR & & & NR \\
\hline Psychoda talamanca & & & & & & & & NR & NR & NR & & NR & PR \\
\hline Psychoda varablanca & NR & & $\mathrm{TL}$ & & & & & NR & & & & NR & NR \\
\hline Total of new records & 5 & 1 & 0 & 0 & 1 & 1 & 1 & 10 & 5 & 6 & 1 & 7 & 7 \\
\hline
\end{tabular}

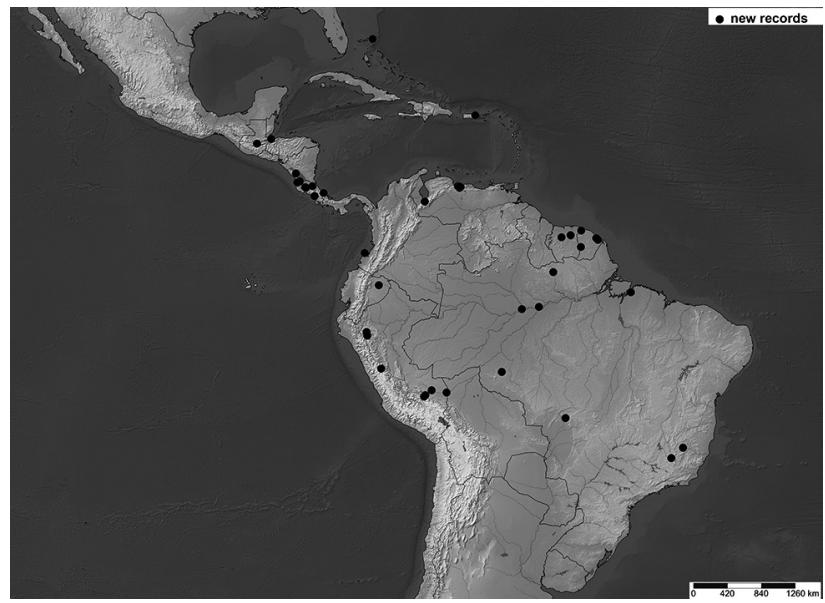

Figure 1. A: map showing points of new records for countries or states/provinces territories.

The list of geographical records for the species treated here is organized by countries from north to south, west to east. Countries are given in Capital letters. The localities of the records are according to the original labels of specimens (transcribed literally in the text), including the coordinates, when available. Exception is data of altitude where 'm', meters, was replaced by 'masl', meters above sea level. Map with points of new records was produced on SimpleMappr tool (Shorthouse, 2010).

\section{RESULTS AND DISCUSSION}

Bellow it is listed the first record to Brazil of seven species and one genus. The complete known distribution of these species is presented along with new records of Psychodinae species for other 10 countries of the Neotropical region, namely Guatemala, Nicaragua, Bahamas, Puerto Rico, Colombia, Venezuela, Surinam, French Guiana, Ecuador and Peru (Table 1, Fig. 1). The distribution of 13 species already known from Brazilian territory is also updated, with new records for the states
Table 2. Species with new records for states in Brazil and their known distribution in the country. Brazilian states are abbreviated with 2-letter codes. $\mathrm{NR}=$ new record, $\mathrm{PR}=$ previous record.

\begin{tabular}{|c|c|c|c|c|c|c|c|c|c|c|}
\hline & AM & RO & AP & PA & BA & MT & MG & ES & SP & PR \\
\hline Alepia ancylis & NR & & & & & & & & & \\
\hline Caenobrunettia subditicia & $P R$ & & & NR & & & & & & \\
\hline Eurygarka sp. & & NR & & & & & & & & \\
\hline Platyplastinx culmosus & & NR & & & & & & & & \\
\hline Psychoda amazonensis & $P R$ & & NR & & & & & & & \\
\hline Psychoda atlantica & & & & & & & NR & PR & & \\
\hline Psychoda buxoides & $P R$ & NR & & $P R$ & & PR & & & & \\
\hline Psychoda dantilandensis & & & & & PR & & NR & & & \\
\hline Psychoda entolopha & NR & NR & & & & & NR & & & \\
\hline Psychoda flagellata & & NR & & & & & NR & & & \\
\hline Psychoda laticaula & & NR & & & PR & & & & & \\
\hline Psychoda litotes & $P R$ & & & & & NR & & & & \\
\hline Psychoda obeliske & & & & NR & & & & & & \\
\hline Psychoda savaiiensis & $P R$ & NR & & PR & $P R$ & $P R$ & NR & PR & & \\
\hline Psychodaserraorobonensis & PR & & & NR & PR & & NR & & PR & PR \\
\hline Psychoda setigera & & & & NR & & & & & & \\
\hline Psychoda silvensis & $P R$ & & & & & & NR & & & \\
\hline Psychoda talamanca & $P R$ & NR & & & & & & & & \\
\hline Psychoda trilobatula & $P R$ & NR & & PR & & & & & & \\
\hline Psychoda varablanca & & NR & & & & NR & & & & \\
\hline Psychoda zetoscota & & NR & & PR & PR & & & & & PR \\
\hline Total of new records & 2 & 11 & 1 & 4 & 0 & 2 & 7 & 0 & 0 & 0 \\
\hline
\end{tabular}

of Rondônia, Amapá, Pará, Mato Grosso and Minas Gerais (Table 2, Fig. 1).

\section{Alepia ancylis Quate \& Brown}

Alepia ancylis Quate \& Brown, 2004: 56, figs. 141-143 (Figs. 2A-2B).

New records (determined by L. Quate, confirmed by D. Cordeiro): VENEZUELA, Aragua, $17 \mathrm{~km} \mathrm{~S}$ of Choronf, 760 masl. ECUADOR, Napo, Yasuni Research Station Rios Tivacunu \& Tiputini, 250 masl, $00^{\circ} 38^{\prime} \mathrm{S}, 76^{\circ} 36^{\prime} \mathrm{W}$. BRAZIL, Amazonas, Itacoatiara-Itapiranga $23 \mathrm{~km}$, primary forest, $<100$ masl, $03^{\circ} 03.0^{\prime} \mathrm{S}, 58^{\circ} 43.5^{\prime} \mathrm{W}$; Amazonas, Manacapura $74 \mathrm{~km}$ WSW of Manaus, $03^{\circ} 17^{\prime} 48.8^{\prime \prime} \mathrm{S}, 60^{\circ} 37^{\prime} 38.0^{\prime \prime} \mathrm{W}$; Rondônia, Cacaulândia, $200 \mathrm{~km}$ SSE of Porto Velho, 140 masl, $10^{\circ} 18.0^{\prime} \mathrm{S}, 62^{\circ} 52.1^{\prime} \mathrm{W}$.

Previous record (Quate \& Brown, 2004): PERU, Cuzco Dept., Rio Madre de Dios, $28 \mathrm{~km}$ ESE of Boca Manu, 250 masl, $12^{\circ} 21^{\prime} \mathrm{S}, 70^{\circ} 42^{\prime} \mathrm{W}$ (type locality).

Remarks: This species was described by Quate \& Brown (2004) but illustrations were restricted to male and female genitalia. Herein it is provided photographs of male head, palpus, antennae and wing.

\section{Caenobrunettia sarculosa Quate}

Caenobrunettia sarculosa Quate, 1999: 426-427, figs. 5A-G; Quate \& Brown, 2004: 102, figs. 262-263. 
New record (determined by D. Cordeiro): COLOMBIA, Cauca Prov., Gorgona, alta El Mirador, 180 masl.

Previous records (Quate, 1999; Quate \& Brown, 2004): PANAMA, Barro Colorado I. (type locality). COSTA RICA, Guanacaste, 3 km SE Río Naranjo; Puntarenas, Est. Biol. Las Alturas, $08^{\circ} 57.23^{\prime} \mathrm{N}, 82^{\circ} 50.22^{\prime} \mathrm{W}, 1,550$ masl; Monteverde, 1,500 masl. SURINAM, Brownsberg Nature Park, 100 km S Paramaribo, $04^{\circ} 57^{\prime} \mathrm{N}, 55^{\circ} 11^{\prime} \mathrm{W}, 300-450$ masl; Raleighvallen, $170 \mathrm{~km}$ SW Paramaribo, $04^{\circ} 43^{\prime} \mathrm{N}, 56^{\circ} 12^{\prime} \mathrm{W}, 70$ masl. BRAZIL, Amazonas, Manacapuru-Novo Airão, km 46-50, 02 ${ }^{\circ} 59.3^{\prime} \mathrm{S}$, $60^{\circ} 53.6^{\prime} \mathrm{W}$, 50 masl. PERU, Cusco, Río Madre de Dios, $28 \mathrm{~km}$ ESSE Boca Manu, $12^{\circ} 21^{\prime} \mathrm{S}, 70^{\circ} 42^{\prime} \mathrm{W}, 25$ masl.

\section{Caenobrunettia subditicia Quate \& Brown}

Caenobrunettia subditicia Quate \& Brown, 2004: 102-104, fig. 264.

New record (determined by L. Quate, confirmed by D. Cordeiro): BRAZIL, Pará, Belém.

Previous records (Quate \& Brown, 2004): BRAZIL, Amazonas, Manacapuru-Novo Airão, $02^{\circ} 59.3^{\prime}$ S, $60^{\circ} 53.6^{\prime} \mathrm{W}$ (type locality). SURINAM, Raleighvallen, $170 \mathrm{~km} \mathrm{SW}$ of Paramaribo, 70 masl, $04^{\circ} 43^{\prime} \mathrm{N}, 56^{\circ} 12^{\prime} \mathrm{W}$; SURINAM, Brownsberg Nature Park, 100 km S of Paramaribo, pri-

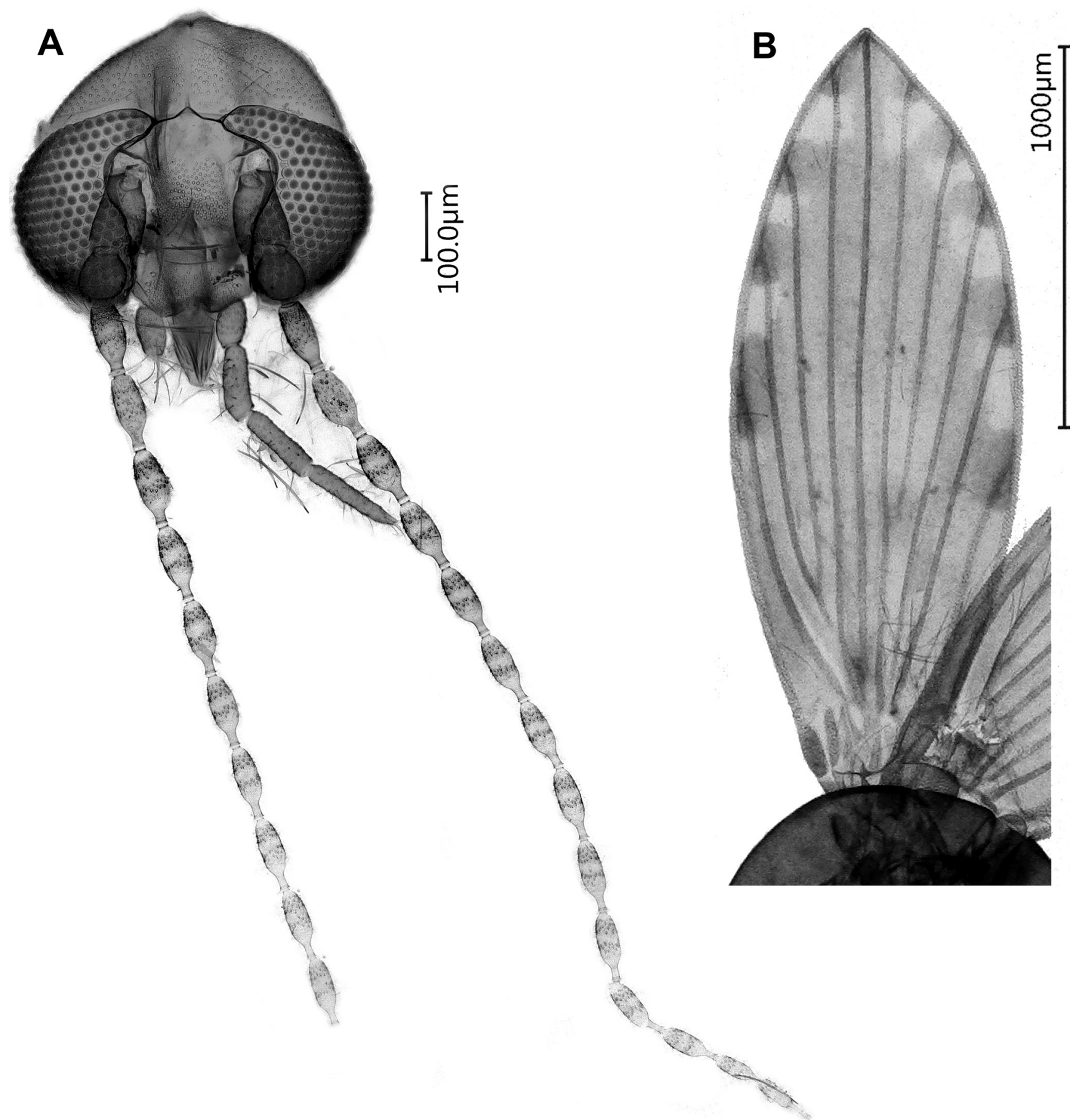

Figure 2. Alepia ancylis Quate \& Brown, male paratype from Peru. (A) Head, palpus and complete antennae. (B) Wing. 
mary forest, $300-450$ masl, $04^{\circ} 57^{\prime} \mathrm{N}, 55^{\circ} 11^{\prime} \mathrm{W}$. FRENCH GUIANA, Maripasoula.

\section{Eurygarka sp. \\ (Figs. 3A-3F)}

New record for the genus (determined by D. Cordeiro): BRAZIL, Rondônia, Cacaulândia, 200 km SSE of Porto Velho, 22-31.x.97, 140 masl, 10¹8.0'S, 6252.1'W, col. W.J. Hanson (LACMENT 268194).

Remarks: This female specimen is undoubtfully an Eurygarka for the following characters: eye bridge with four rows of facets, divided by one facet diameter; palp segments two and three slightly swollen; palp segments 1-3 each with transverse, ovoid sensory organ placed mid-length on their lateral surface; first palp segment a little greater than $1 / 2$ the length of second, distal three palp segments slightly increasing in length; female cerci short; hypovalvae with single median protuberance, digitiform (Curler \& Moulton, 2008).

This is the first record of this genus to Brazil. This is possibly the female of Eurygarka freyrei Jezek et al., 2011 (described from Bolivia), but its morphology is also similar to the male of E. cyphostylus Curler, 2008 (from the USA), so the association is dubious. The characters that link this female to the males of these two species are: distance of eyes, number of facet rows, the shape of frons groups of setae, the shape of palpus, the shape of Sc vein on the wing and infuscation of costal cell.

\section{Platyplastinx culmosus Quate \& Brown}

Platyplastinx culmosus Quate \& Brown, 2004: 67, figs. 164-166.

New record (determined by L. Quate, confirmed by D. Cordeiro): BRAZIL, Rondônia, Cacaulândia, 200 km SSE of Porto Velho, 140 masl, $10^{\circ} 18.0^{\prime} \mathrm{S}, 62^{\circ} 52.1^{\prime} \mathrm{W}$.

Previous record (Quate \& Brown, 2004): ECUADOR, Napo, Yasuni Research Station, Río Tivacunu \& Tiputini, 250 masl, $00^{\circ} 38^{\prime} \mathrm{S}, 76^{\circ} 36^{\prime} \mathrm{W}$ (type locality).

\section{Psychoda amazonensis Cordeiro \& Bravo}

Psychoda amazonensis Cordeiro \& Bravo, 2008: 116, figs. 1-14; Cordeiro et al., 2011: 7 (Fig. 4).

New record (determined by D. Cordeiro): BRAZIL, Amapá (no locality).

Previous records (Cordeiro \& Bravo, 2008): BRAZIL, Amazonas, Silves, Saracá (type locality).

Remarks: This species is known to be viviparous, as females are captured with many first instar larvae inside the abdômen (Fig. 4). It can be easily identified by the characteristic hypovalvae (indicated by an arrow on figure 4) and short cerci of the female.

\section{Psychoda atlantica Cordeiro, Bravo \& Carvalho}

Psychoda atlantica Cordeiro, Bravo \& Carvalho, 2011: 18-19, figs. 44-53.

New record (determined by D. Cordeiro): BRAZIL, Minas Gerais, Parque Est. do Rio Doce $240 \mathrm{~km}$ E of Belo Horizonte, 350 masl, $18^{\circ} 45.18^{\prime} \mathrm{S}, 42^{\circ} 38.00^{\prime} \mathrm{W}$.

\section{Previous records (Cordeiro et al., 2011; Shimabukuro} et al., 2016): BRAZIL, Espírito Santo, 19¹3'10.5"S, $40^{\circ} 46^{\prime} 23.8^{\prime \prime} \mathrm{W}$ (type locality); Espírito Santo, 19¹2'54.8' $\mathrm{S}$, 4047'52.5"W; São Paulo, Ilha do Cardoso; Paraná, Antonina, res. Sapitanduva.

\section{Psychoda buxoides Quate}

Psychoda buxoides Quate, 1996: 71, figs. 28a-e; Quate, 1999: 439; Cordeiro et al., 2011: 8, figs. 1-8.

New records (determined by D. Cordeiro): GUATEMALA, Izabal, Puerto Barrios, 10 masl. COSTA RICA, Heredia, Puerto Viejo de Sarapaqui, Est. Biol. LaSelva, 50-100 masl, LN-268.000-535.600; Limon, Puerto Viejo deTalamanca, sea level, LS-401.000-600.000. VENEZUELA, Aragua, Maracay. SURINAM, Raleighvallen, $170 \mathrm{~km}$ SW of Paramaribo, 70 masl, $04^{\circ} 43^{\prime} \mathrm{N}, 56^{\circ} 12^{\prime} \mathrm{W}$. PERU, Cuzco, Rio Madre de Dios, $28 \mathrm{~km}$ ESE of Boca Manu, 250 masl, $12^{\circ} 21^{\prime} \mathrm{S}, 70^{\circ} 42^{\prime} \mathrm{W}$. BRAZIL, Rondônia, Cacaulândia, 200 km SSE of Porto Velho, 140 masl, $10^{\circ} 18.0^{\prime} \mathrm{S}, 62^{\circ} 52.1^{\prime} \mathrm{W}$; Mato Grosso, Chapada dos

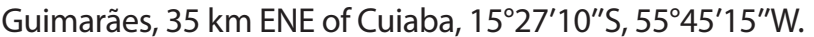

Previous records (Quate, 1996, 1999; Cordeiro et al., 2011): COSTA RICA, Guanacaste, La Pacifica, 40 km SE of Liberia, 100 masl (type locality); Guanacaste, Parq. Nac. Santa Rosa, Rio Cuajiminquil, 280 masl, 313400(N) 358900(E); Guanacaste, Parque Nac. Santa Rosa, sea level; Guanacaste, Estacion Pitilla, 9 km S Santa Cecilia, 700 masl. PANAMA, Canal Zone, Barro Colorado Is., \#984, $09^{\circ} 09^{\prime} \mathrm{N}, 79^{\circ} 51^{\prime} \mathrm{W}$. BRAZIL, Amazonas, ManacapuruNovo Airao km 46-50, 0259.0'S, 6053.6'W; Amazonas, Manacapura $74 \mathrm{~km}$ WSW of Manaus, $03^{\circ} 17^{\prime} 48.8^{\prime \prime} \mathrm{S}$, $60^{\circ} 37^{\prime} 38.0^{\prime \prime} \mathrm{W}$; Amazonas, Pitinga, $258 \mathrm{~km} \mathrm{~N}$ of Manaus, Bica, $00^{\circ} 45^{\prime} 21^{\prime \prime} \mathrm{S}, 60^{\circ} 04.00^{\prime} \mathrm{W}$; Amazonas, Silves, Saracá; Amazonas, Manaus, Res. Ducke; Pará, Estrada do Caripi km 4, 15 km SW of Belem; Pará, Chácara N.S. Nazaré; Mato Grosso, Barão de Melgaço, baia de Bracoruré.

\section{Psychoda dantilandensis Bravo, Cordeiro \& Chagas}

Psychoda dantilandensis Bravo, Cordeiro \& Chagas, 2006: 8-9, figs. 19-27; Cordeiro et al., 2011: 9-11, figs. $9-14$. 


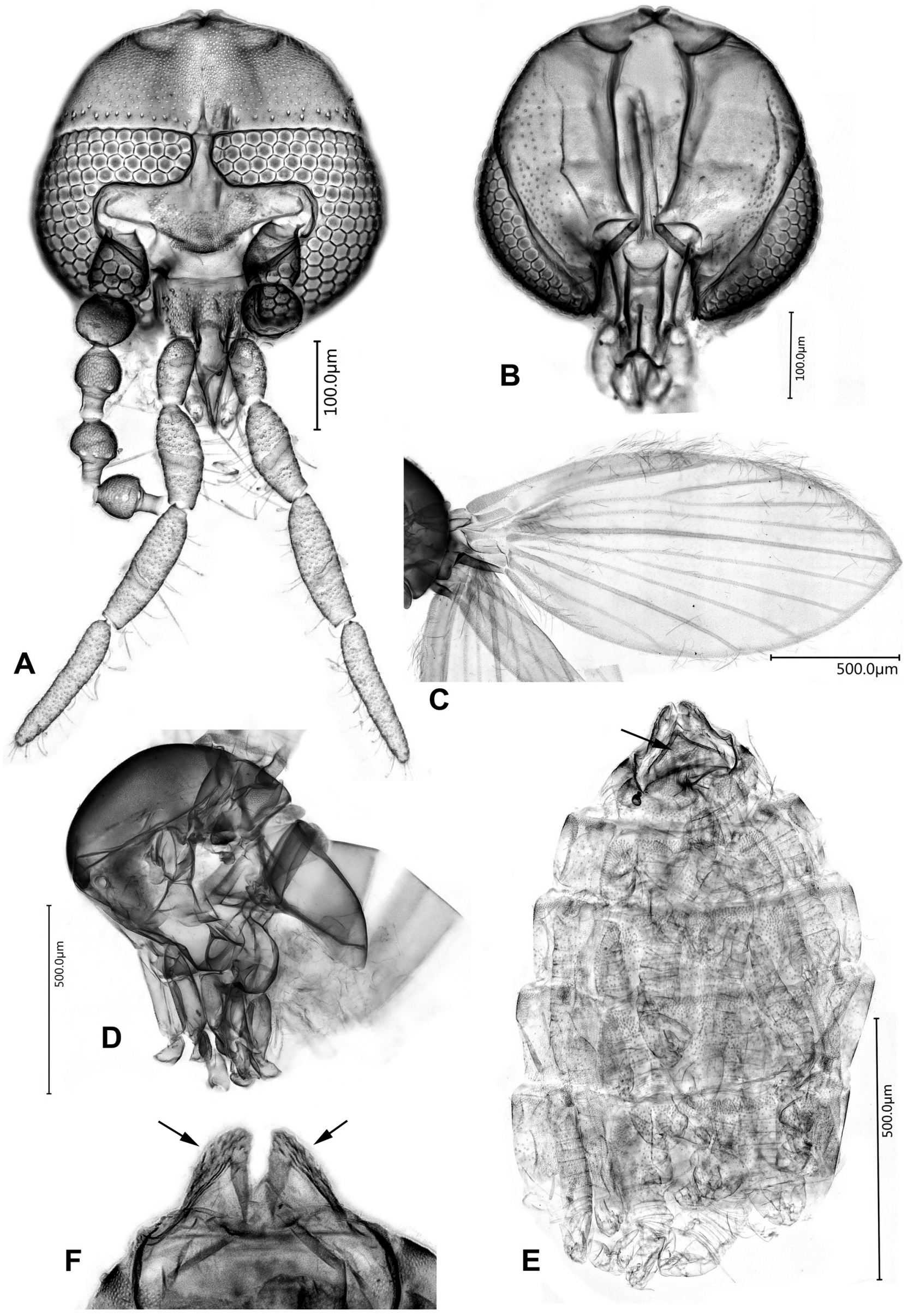

Figure 3. Eurygarka sp., female. (A) Head, anterior view. (B) Head, posterior view. (C) Wing. (D) Thorax, lateral view. (E) Abdomen, ventral view, arrow indicating hypovalvae. (F) Terminalia in detail, dorsal view, arrows indicating cerci. 


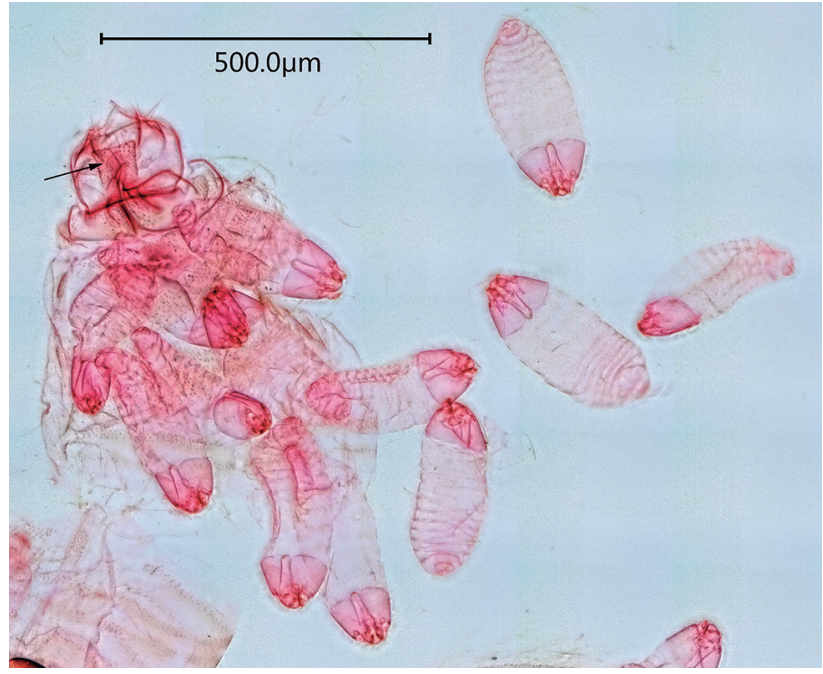

Figure 4. Psychoda amazonensis Cordeiro \& Bravo, female, dissected abdomen showing characteristic hypovalvae (indicated by arrow) on terminalia and many first instar larvae.

New record (determined by $D$. Cordeiro): BRAZIL, Minas Gerais, Parque Est. do Rio Doce 240 km E of Belo Horizonte, $350 \mathrm{msal}, 18^{\circ} 45.18^{\prime} \mathrm{S}, 42^{\circ} 38.00^{\prime} \mathrm{W}$.

Previous records (Bravo et al., 2006): BRAZIL, Bahia, Dantilândia $15^{\circ} 06^{\prime} \mathrm{S}, 40^{\circ} 00 \mathrm{~W}$ (type locality); Bahia, Itabuna, $14^{\circ} 45^{\prime} \mathrm{S}, 39^{\circ} 17^{\prime} \mathrm{W}$.

\section{Psychoda entolopha Quate}

Psychoda entolopha Quate, 1996: 77-79, fig. 31g.

New records (determined by D. Cordeiro): GUATEMALA, Baja Verapaz, La Union Barrios, 1,500 masl. NICARAGUA, Granada, Granada Volcan Mombacho, San Joaquin, 1,100 masl, $11^{\circ} 50^{\prime} \mathrm{N}, 85^{\circ} 51^{\prime} \mathrm{W}$. COSTA RICA, Guanacaste, Volcan Cacao, E of SR, 1,000-1,100 masl, 323200N, 375600E; Guanacaste, Estacion Pitilla, 9 km S Santa Cecilia, 700 masl; Guanacaste, Pr., Montevere, 1,500 masl. VENEZUELA, Barinas, La Azulita, 1,400 masl, $08^{\circ} 42^{\prime} \mathrm{N}$, $71^{\circ} 28^{\prime} \mathrm{W}$; Aragua, $20 \mathrm{~km} \mathrm{~S}$ of Choronf, 880 masl; Aragua, $19 \mathrm{~km} \mathrm{~N}$ of Maracay, 1,280 masl; Aragua, $12 \mathrm{~km} \mathrm{NW} \mathrm{El}$ Limon, 1,020 masl; Aragua, Maracay. PERU, Huanuco, Huanuco, Higueras, 2,000 masl; Cuzco, Rio Madre de Dios, $28 \mathrm{~km}$ ESE of Boca Manu, 250 masl, $12^{\circ} 21^{\prime} \mathrm{S}, 70^{\circ} 42^{\prime} \mathrm{W}$. BRAZIL, Amazonas, Manacapura 74 km WSW of Manaus, 0317'48.8'S, 60³7'38.0"W; Rondônia, Cacaulândia, $200 \mathrm{~km} \mathrm{SSE}$ of Porto Velho, 140 masl, $10^{\circ} 18.0^{\prime} \mathrm{S}, 62^{\circ} 52.1^{\prime} \mathrm{W}$; Minas Gerais, Parque Est. do Rio Doce $240 \mathrm{~km}$ E of Belo Horizonte, 350 masl, $18^{\circ} 45.18^{\prime} \mathrm{S}, 42^{\circ} 38.00^{\prime} \mathrm{W}$; Minas Gerais, University Biol Reserve Belo Horizonte, $19^{\circ} 55^{\prime} \mathrm{S}, 43^{\circ} 56^{\prime} \mathrm{W}$.

Previous records (Quate, 1996): COSTA RICA, Heredia, S Rafael de Vara Blanca Rio Santo Domingo, 1,700 masl (type locality); Heredia, Vara Blanca, 1,800 masl, LN-239.500-519.000; San José, 2 km W of Empalme, 2,300 masl; San José, San Gerardo de Dota Alb. Savegre, 2,250 masl, $09^{\circ} 33^{\prime} 07^{\prime \prime} \mathrm{N}, 83^{\circ} 48^{\prime} 30^{\prime \prime} \mathrm{E}$; Cartago, $30 \mathrm{~km} \mathrm{~N}$ of
San Isidro, 2,640 masl, 09³2.7' N, 8342.8' $\mathrm{E}$; Puntarenas, Estacion Pittier, $22 \mathrm{~km}$ N of San Vito, 1,670 masl, $09^{\circ} 01.4^{\prime} \mathrm{N}$, $82^{\circ}$ 57.5'E; Puntarenas, Estación Pittier, 20 km N of San Vito, 1,800 masl; Puntarenas, Est. Biol. Las Alturas Coto Brus, 1,550 masl, $08^{\circ} 57^{\prime} 14^{\prime \prime} \mathrm{N}, 82^{\circ} 50^{\prime} 13^{\prime \prime} \mathrm{E}$; Puntarenas, $18 \mathrm{~km} N$ of San Isidro, 1,700 masl; Puntarenas, $13 \mathrm{~km} \mathrm{~N}$ of San Isidro, 1,500 masl; Puntarenas, Monteverde Est. Biol. Monteverde, 1,550 masl, LN-255700(N), 448700(E); Limón, Puerto Viejo de Tal., mixed forest, 0-100 masl.

\section{Psychoda flagellata Quate}

Psychoda flagellata Quate, 1996: 79, fig. 31f; Quate, 1999: 440; Collantes \& Martinez-Ortega, 1999: 21.

New records (determined by $D$. Cordeiro): COSTA RICA, Guanacaste, Estacion Pitilla, 9 km S Santa Cecilia, 700 masl; Guanacaste, Parq. Nac. Santa Rosa, evergreen forest, 280 masl, 313400(N) 358900(E). SURINAM, Brownsberg Nature Park, $100 \mathrm{~km} \mathrm{~S}$ of Paramaribo, 300-450 masl, $04^{\circ} 57^{\prime} \mathrm{N}, 55^{\circ} 11^{\prime} \mathrm{W}$. FRENCH GUIANA, 37 km S of Cayenne, 50-100 masl; Maripasoula. BRAZIL, Rondônia, Cacaulândia, $200 \mathrm{~km}$ SSE of Porto Velho, 140 masl, 10¹8.0'S, 62²5.1'W; Minas Gerais, Parque Est. do Rio Doce 240 km E of Belo Horizonte, 350 msal, $18^{\circ} 45.18^{\prime} \mathrm{S}, 42^{\circ} 38.00^{\prime} \mathrm{W}$.

Previous records (Quate, 1996, 1999; Collantes \& Martinez-Ortega, 1999): COSTA RICA, Heredia, Puerto Viejo de Sarapaqui Est. Biol LaSelva, 50-100 masl, LN-268.000-535.600 (type locality). Limon, Puerto Viejo de Talaman., sea level, LS-401.000-600.000; Limon, Puerto Viejo de Tal., sea level, $09^{\circ} 39.4^{\prime} \mathrm{N}, 85^{\circ} 45.9^{\prime} \mathrm{W}$; Limon, Res. Biol. Hitoy Cerere, R.Cerere, shaded stream, 100-200 masl, $09^{\circ} 48.4^{\prime} \mathrm{N}, 83^{\circ} 01.5^{\prime} \mathrm{W}$. NICARAGUA, León; Masaya; Rio San Juan; Zelaya. PANAMA, Canal Zone, Barro Colorado Is., \#986, $09^{\circ} 09^{\prime} \mathrm{N}, 79^{\circ} 51^{\prime} \mathrm{W}$; San Blas, Nusagandi Reserve \#2046, $09^{\circ} 20^{\prime} \mathrm{N}, 79^{\circ} 00^{\prime} \mathrm{W}$.

\section{Psychoda laticaula Quate}

Psychoda laticaula Quate, 1996: 67, fig. 26f; Collantes \& Martinez-Ortega, 1999: 22; Cordeiro et al., 2011:11-13, figs. 15-22.

New records (determined by D. Cordeiro): COSTA RICA, Puntarenas, $14 \mathrm{~km}$ SW of San Isidro, 880 masl, $09^{\circ} 19.0^{\prime} \mathrm{N}$, $83^{\circ} 46.5^{\prime} \mathrm{W}$; Puntarenas, $18 \mathrm{~km}$ N of San Isidro, 1,700 masl; Puntarenas, Estación Pittier, 20 km N of San Vito, streamside, 1,800 masl; Puntarenas, Estación Pittier, 22 km N of San Vito, 1,670 masl; Puntarenas, Estación Pitilla, 9 km S Santa Cecilia, 700 masl. VENEZUELA, Barinas, La Azulita, 1,400 masl, $08^{\circ} 42^{\prime} \mathrm{N}, 71^{\circ} 28^{\prime} \mathrm{W}$; Aragua, $20 \mathrm{~km} \mathrm{~S}$ of Choronf, 880 masl; Aragua, $22 \mathrm{~km} \mathrm{~S}$ of Choronf, 1,000 masl; Aragua, $19 \mathrm{~km} \mathrm{~N}$ of Maracay, 1,280 masl; Aragua, 15 km NW El Limon. PERU, Cuzco, NE of Pilcopata Amazonia Ldg., 450 masl; Cuzco, 26 km W of Pilcopata, cloud forest, 1,500 masl, $13^{\circ} 03.3^{\prime \prime} \mathrm{S}, 71^{\circ} 32.8^{\prime} \mathrm{W}$; Cuzco, 
Rio Madre de Dios, 28 km ESE of Boca Manu, 250 masl, $12^{\circ} 21^{\prime} \mathrm{S}, 70^{\circ} 42^{\prime} \mathrm{W}$; Madre de Dios, Manu Lodge, 300 masl; Amazonas, Chachapoyas, 2,150 masl, 06 ${ }^{\circ} 15^{\prime \prime} \mathrm{S}, 77^{\circ} 53^{\prime} \mathrm{W}$;

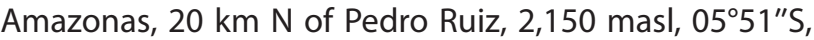
$77^{\circ} 58^{\prime}$ W. BRAZIL, Rondônia, Cacaulândia, 200 km SSE of Porto Velho, 140 masl, $10^{\circ} 18.0^{\prime} \mathrm{S}, 62^{\circ} 52.1^{\prime} \mathrm{W}$.

Previous records (Quate, 1996; Collantes \& MartinezOrtega, 1999; Cordeiro et al., 2011): COSTA RICA, Limon, Puerto Viejo de Talaman, sea level, LS-401.000-600.000 (type locality). Limon, Res. Biol. Hitoy Cerere, Rio Cerere, $100-200$ masl, $09^{\circ} 48.4^{\prime} \mathrm{N}, 83^{\circ} 01.5^{\prime} \mathrm{W}$; Limon, Puerto Viejo de Tal., sea level, $09^{\circ} 39.4^{\prime} \mathrm{N}$, 8545.9'W; Heredia, S Rafael de Vara Blanca Rio Santo Domingo; Heredia, Puerto Viejo de Sarapaqui, Est. Biol. LaSelva, 50-100 masl, LN-268.000-535.600. NICARAGUA, Matagalpa: KM; Rio San Juan: SCA. BRAZIL, Bahia, Ituberá, Res. Michelin.

\section{Psychoda litotes Quate}

Psychoda litotes Quate, 1996: 64-65, figs. 25d-f; Collantes \& Martinez-Ortega, 1999: 22; Cordeiro et al., 2011: 13, figs. 23-31.

New records (determined by D. Cordeiro): VENEZUELA, Barinas, La Azulita, 1,350 masl, $08^{\circ} 42^{\prime} \mathrm{N}, 71^{\circ} 28^{\prime} \mathrm{W}$. FRENCH GUIANA, Maripasoula; 37 km S of Cayenne, 50-100 masl; near St. Laurent de Maroni, $13 \mathrm{~km}$ W of Mara Coastal Woods, 50-100 masl. BRAZIL, Mato Grosso, Chapada dos Guimaraes $35 \mathrm{~km}$ ENE of Cuiaba, $15^{\circ} 27^{\prime} 10^{\prime \prime} \mathrm{S}, 55^{\circ} 45^{\prime} 15^{\prime \prime} \mathrm{W}$.

Previous records (Quate, 1996; Collantes \& MartinezOrtega, 1999; Cordeiro et al., 2011): COSTA RICA, Limon, Puerto Viejo de Tal, sea level, $09^{\circ} 39.4^{\prime} \mathrm{N}, 82^{\circ} 45.9^{\prime} \mathrm{W}$ (type locality); Limon, Res.Biol. Hitoy Cerere, primary forest, $100-200$ masl, $09^{\circ} 48.4^{\prime} \mathrm{N}, 83^{\circ} 01.5^{\prime} \mathrm{W}$; Heredia, Puerto Viejo de Sarapaqui Est. Biol LaSelva, 50-100 masl, LN-268.000-535.600; Guanacaste, Estacion Pitilla, 9 km S Santa Cecilia, 700 masl; San José, 2 km W of Empalme, 2,300 masl. NICARAGUA, Carazo; Rio San Juan, Se of San Carlos, lowland rain forest, 30 masl, $10^{\circ} 58^{\prime} \mathrm{N}$, $84^{\circ} 20^{\prime}$ W. BRAZIL, Amazonas, Silves, saracá; Amazonas, Manacapuru, Cajatuba.

\section{Psychoda obeliske Quate, 1996}

Psychoda obeliske Quate, 1996: 65, figs. 25g-h; Collantes \& Martinez-Ortega, 1999: 22.

New records (determined by D. Cordeiro): GUATEMALA, Izabal, Puerto S Tomas Castillo, 10 masl. PUERTO RICO, Luquillo, Caribbean National Forest, El Verde Exp. Station, 350 masl, $18^{\circ} 19.3^{\prime} \mathrm{N}, 65^{\circ} 49.6^{\prime} \mathrm{W}$. VENEZUELA, Barinas, La Azulita, 1,350 masl, $08^{\circ} 42^{\prime} \mathrm{N}$, $71^{\circ} 28^{\prime}$ W. SURINAM, Brownsberg Nature Park, $100 \mathrm{~km}$ S of Paramaribo, 450 masl, $04^{\circ} 57^{\prime} \mathrm{N}, 55^{\circ} 11^{\prime} \mathrm{W} ; 170 \mathrm{~km} \mathrm{SW}$ of Paramaribo, 70 masl, $04^{\circ} 43^{\prime} \mathrm{N}, 56^{\circ} 12^{\prime} \mathrm{W}$. FRENCH GUIANA, 65 km S of Cayenne, 50-100 masl; 17 km E of St. Laaurent de Maroni, sea level; 37 km S of Cayenne, 50-100 masl. PERU, Cuzco, Rio Madre de Diso, 28 km ESE of Boca Manu, 250 masl, $12^{\circ} 21^{\prime} \mathrm{S}, 70^{\circ} 42^{\prime} \mathrm{W}$; Cuzco, $26 \mathrm{~km}$ W of Pilcopata, forest edge, 1,500 masl, $13^{\circ} 03^{\prime} \mathrm{S}, 71^{\circ} 32^{\prime} \mathrm{W}$. BRAZIL, Pará, Serra do Cachorro, $395 \mathrm{~km}$ NW of Manaus, $00^{\circ} 49.8^{\prime} 00^{\prime \prime} \mathrm{N}$, $57^{\circ} 07.0^{\prime} 09^{\prime \prime} \mathrm{W}$; Rondônia, Cacaulândia, 200 km SSE of Porto Velho, 140 masl, $10^{\circ} 18.0^{\prime} \mathrm{S}, 62^{\circ} 52.1^{\prime} \mathrm{W}$.

Previous records (Quate, 1996; Collantes \& Martinez-Ortega, 1999): COSTA RICA, Heredia, Puerto Viejo de Sarapaqui Est. Biol LaSelva, 50-100 masl, LN-268.000-535.600 (type locality). NICARAGUA, Carazo; Chinadega; Rio San Juan.

\section{Psychoda savaiiensis Edwards, 1928}

Psychoda savaiiensis Edwards, 1928: 74; Quate, 1962: 63; Duckhouse, 1973: 13; Quate, 1996: 71; Quate, 1999: 439; Collantes \& Martinez-Ortega, 1999: 23; Cordeiro et al., 2011: 14-15.

Psychoda rarotongensis Satchell, 1953: 183; Quate, 1955: 208; Quate, 1959: 213-214.

Psychoda lucia Quate, 1954: 349-350, figs. 28-31.

New records (determined by D. Cordeiro): GUATEMALA, Izabal, Puerto Barrios, 10 masl. BAHAMAS, Abaco Is., Treasure Cay, broadleaf forest, sea level, $26^{\circ} 49.8^{\prime} \mathrm{N}$, 77¹7.3'W. VENEZUELA, Aragua, 12 km NW El Limon, 1,020 masl; Aragua, Henri Pittier National Park, Rancho Grande, 1,100 masl, $10^{\circ} 20^{\prime} \mathrm{N}, 67^{\circ} 41^{\prime} \mathrm{W}$; Aragua, $25 \mathrm{~km} \mathrm{NW}$ of Maracay Aponte, 50 masl, $10^{\circ} 25^{\prime} \mathrm{N}, 67^{\circ} 46^{\prime} \mathrm{W}$; Barinas, El Tao, La Azulita, 1,350 masl, $08^{\circ} 41.7^{\prime} \mathrm{N}, 71^{\circ} 27.8^{\prime} \mathrm{W}$. SURINAM, Raleighvallen, $170 \mathrm{~km}$ SW of Paramaribo, 70 masl, $04^{\circ} 43^{\prime} \mathrm{N}$, 56 $12^{\prime} \mathrm{W}$; Brownsberg Nature Park, $100 \mathrm{~km} \mathrm{~S}$ of Paramaribo, 450 masl, $04^{\circ} 57^{\prime} \mathrm{N}, 55^{\circ} 11^{\prime} \mathrm{W}$. FRENCH GUIANA, $30 \mathrm{~km} S$ of St. Laurent de Maroni, 0-100 masl; near St. Laurent de Maroni, $13 \mathrm{~km}$ W of Mara Costal Woods, 50-100 masl; Maripasoula; $37 \mathrm{~km} \mathrm{~S}$ of Cayenne, 50-100 masl; 65 km S of Cayenne, 50-100 masl. PERU, Madre de Dios, $5 \mathrm{~km}$ E of Puerto Maldonado, L Sandoval, 300 masl, $12^{\circ} 36^{\prime} \mathrm{S}, 6^{\circ} 02^{\prime} \mathrm{W}$. BRAZIL, Rondônia, Cacaulândia, 200 km SSE of Porto Velho, 140 masl, $10^{\circ} 18.0^{\prime} \mathrm{S}, 62^{\circ} 52.1^{\prime} \mathrm{W}$; Minas Gerais, Parque Est. do Rio Doce $240 \mathrm{~km}$ E of Belo Horizonte, 350 masl, $18^{\circ} 45.18^{\prime} \mathrm{S}$, $42^{\circ} 38.00^{\prime} \mathrm{W}$.

Previous records on the Neotropics (this species has a pantropical distribution) (Quate, 1959, 1996, 1999; Collantes \& Martinez-Ortega, 1999; Cordeiro et al., 2011): NICARAGUA, Granada, Volcán Mombacho El Progresso; Granada, Volcan Mombacho San Joaquin, 1,100 masl, $11^{\circ} 50^{\prime} \mathrm{N}, 85^{\circ} 51^{\prime} \mathrm{W}$; Rio San Juan, SE of San Carlos, lowland rain forest, 30 masl, $10^{\circ} 58^{\prime} \mathrm{N}, 84^{\circ} 20^{\prime} \mathrm{W}$; Zelaya, Las Americas; Carazo; Chinadega; Matagalpa. COSTA RICA, Guanacaste, La Pacifica, 40 km SE of Liberia, 100 masl; Guanacaste, Parque Nac. Santa Rosa, sea level; Guanacaste, Estacion Pitilla, 9 km S Santa Cecillia, 700 masl; Heredia, Puerto Viejo de Sarapaqui Est Biol LaSelva, 50-100 masl, LN-268.000-535.600; Limon, 
Puerto Viejo de Talaman, sea level, LS-401.000-600.000; Puntarenas, 14 km SW of San Isidro, 880 masl; Higuito, San Mateo; San Pedro de Montes de Oca. PANAMA, Canal Zone, Barro Colorado Is., $09^{\circ} 09^{\prime} \mathrm{N}, 7^{\circ} 51^{\prime} \mathrm{W}$. PUERTO RICO, Luquillo, Caribbean National Forest, El Verde Exp. Station, 350 masl, $18^{\circ} 19.3^{\prime} \mathrm{N}, 65^{\circ} 49.6^{\prime} \mathrm{W}$; El Semil, Villalba. BRAZIL, Amazonas, Manacapuru 74 km WSW of Manaus, $03^{\circ} 17^{\prime} 48^{\prime \prime} \mathrm{S}, 60^{\circ} 37^{\prime} 38^{\prime \prime} \mathrm{W}$; Amazonas, Pitinga, $258 \mathrm{~km} \mathrm{~N}$ of Manaus, $00^{\circ} 45^{\prime} 21^{\prime \prime} \mathrm{S}, 60^{\circ} 04^{\prime} 00^{\prime \prime} \mathrm{W}$; Amazonas, ItacoatiaraItapiranga km 23, < 100 masl, 0303.0'S, 5843.5'W; Pará, Estrada do Caripi km 4, 15 km SW of Belem; Pará, Serra do Cachorro $395 \mathrm{~km}$ NW of Manaus, 395 masl, $00^{\circ} 49.8^{\prime} \mathrm{N}$, $57^{\circ} 07^{\prime} 9^{\prime \prime}$ W; Pará, Santarém, Ch. N.S. Nazaré; Mato Grosso, Chapada dos Guimaraes 35 km ENE of Cuiaba, $15^{\circ} 27^{\prime} 10^{\prime \prime} \mathrm{S}, 55^{\circ} 45^{\prime} 15^{\prime \prime} \mathrm{W}$; Mato Grosso, Barão de Melgaço, baia de Brocoruré; Bahia, Sauípe; Bahia, Cachoeira, Faz. Villa Rial; Bahia, Ituberá, Res. Michellin; Espírito Santo, Pancas, Faz. Juliberto Stur; Espírito Santo, Santa Maria de Jetibá; Paraná, Jundiaí.

\section{Psychoda serraorobonensis Bravo, Cordeiro \& Chagas}

Psychoda serraorobonensis Bravo, Cordeiro \& Chagas, 2006: 10-11, figs. 28-34; Cordeiro et al., 2011: 15-16, figs. $32-33$.

New records (determined by $D$. Cordeiro): BRAZIL, Pará, Serra do Cachorro 395 km NW of Manaus, 395 masl,

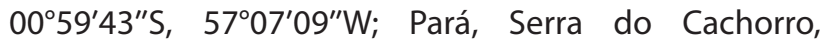
395 km NW of Manaus, 395 masl, 0049.8'S, 5707'9'W; Minas Gerais, Parque Est. do Rio Doce $240 \mathrm{~km}$ E of Belo Horizonte, 350 msal, $18^{\circ} 45.18^{\prime} \mathrm{S}, 42^{\circ} 38.00^{\prime} \mathrm{W}$.

Previous records (Bravo et al., 2006; Cordeiro et al., 2011): BRAZIL, Bahia, Serra do Orobó (type locality); Amazonas, Itacoatiara-Itapiranga km 23, < 100 masl, 0303.0'S, 58 43.5' W; Amazonas, Manacapuru-Novo Airao km 46-50, 0259.0'S, 6053.6’W; Amazonas, Pitinga; Amazonas, Silves, Sacará; Bahia, Senhor do Bonfim, Serra de Santana; Bahia, Cachoeira, Faz. Villa Rial; São Paulo, Ilha do Cardoso; Paraná, Antonina, res. Sapitanduva.

\section{Psychoda setigera Tonnoir}

Psychoda setigera Tonnoir, 1922: 84; Quate, 1955: 202, fig. 63; Quate, 1996: 65-67, figs. 26a-d; Collantes \& Martinez-Ortega, 1999: 23.

New records (determined by D. Cordeiro): VENEZUELA, Aragua, Henri Pittier National Park Rancho Grande, 1,100 masl, $10^{\circ} 20^{\prime} \mathrm{N}, 67^{\circ} 41^{\prime} \mathrm{W}$; Barinas, la Azulita, 2,200 masl, $08^{\circ} 42^{\prime} \mathrm{N}, 71^{\circ} 28^{\prime} \mathrm{W}$. FRENCH GUIANA, St. Jean, $15 \mathrm{~km} \mathrm{~S}$ of St. Laurent de Maroni, sea level; $37 \mathrm{~km} \mathrm{~S}$ of Cayenne, 50-100 masl. BRAZIL, Pará, Serra do Cachorro, $395 \mathrm{~km}$ NW of Manaus, $00^{\circ} 49.8^{\prime} 00^{\prime \prime} \mathrm{N}, 57^{\circ} 07.0^{\prime} 09^{\prime \prime} \mathrm{W}$.

Previous records on the Neotropics (Quate, 1996; Collantes \& Martinez-Ortega, 1999): NICARAGUA,
Granada, Volcan Mombacho, cloud forest, 1,100 masl, $11^{\circ} 50^{\prime} \mathrm{N}, 85^{\circ} 51^{\prime} \mathrm{W}$; Madriz. COSTA RICA, Heredia, S Rafael de Vara Blanca, Rio Santo Domingo, 1,700 masl; Heredia, Vara Blanca, 1,800 masl, LN-239.500-519.000; Heredia, Puerto Viejo de Sarapaqui Est Biol LaSelva, 50-100 masl, LN-268.000-535.600; Guanacaste, Estacion Pitilla, 9 km S Santa Cecillia, 700 masl; Guanacaste, La Pacifica, 40 km SE of Liberia, 100 masl; Guanacaste, Volcan Cacao, E of SR, 1,000-1,100 masl, 323200N, 375600E; Limon, Puerto Viejo de Talaman., sea level, LS-401.000-600.000; San José, $2 \mathrm{~km}$ W of Empalme, 2,300 masl; Cartago, $44 \mathrm{~km} \mathrm{~S}$ of Cartago, 2,350 masl, $09^{\circ} 40.7^{\prime} \mathrm{N}, 83^{\circ} 57.7^{\prime} \mathrm{W}$; Cartago, $30 \mathrm{~km} \mathrm{~N}$ of San Isidro, 2,640 masl, $09^{\circ} 32.7^{\prime} \mathrm{N}, 83^{\circ} 42.8^{\prime} \mathrm{W}$; San José, 2 km W of Empalme.

\section{Psychoda silvensis Cordeiro, Bravo \& Carvalho}

Psychoda silvensis Cordeiro, Bravo \& Carvalho, 2011:31-32, figs. 132-139.

New record (determined by $D$. Cordeiro): BRAZIL, Minas Gerais, University Biol. Reserve Belo Horizonte, $19^{\circ} 55^{\prime} \mathrm{S}, 43^{\circ} 56^{\prime} \mathrm{W}$.

Previous records (Cordeiro et al., 2011): BRAZIL, Amazonas, Silves, Saracá (type locality); Amazonas, Itacoatiara-Itapiranga km 23, < 100 masl, 0303.0'S, $58^{\circ} 43.5^{\prime} \mathrm{W}$

\section{Psychoda talamanca Quate}

Psychoda talamanca Quate, 1996: 77, fig. 31a; Cordeiro et al., 2011: 16-17, figs. 36-43.

New records (determined by D. Cordeiro): VENEZUELA, Barinas, la Azulita, 1,400-1,900 masl, $08^{\circ} 42^{\prime} \mathrm{N}, 71^{\circ} 28^{\prime} \mathrm{W}$; Aragua, $12 \mathrm{~km} \mathrm{NW}$ El Limon, 1,020 masl; Aragua, 10 km N El Limon; Aragua, 17 km S of Choronf, 760 masl; Aragua, Maracay, wooded hillside; Aragua, 19 km N of Maracay, 1,280 masl. SURINAM, Brownsberg Nature Park, 100 km S of Paramaribo, 450 masl, $04^{\circ} 57^{\prime} \mathrm{N}, 55^{\circ} 11^{\prime} \mathrm{W}$. FRENCH GUIANA, $37 \mathrm{~km} \mathrm{~S}$ of Cayenne, 50-100 masl; $17 \mathrm{~km} \mathrm{E} \mathrm{of} \mathrm{St.} \mathrm{Laurent} \mathrm{de}$ Maroni, sea level. PERU, Cuzco, Rio Madre de Dios, $28 \mathrm{~km}$ ESE of Boca Manu, 250 masl, $12^{\circ} 21^{\prime} \mathrm{S}, 70^{\circ} 42^{\prime} \mathrm{W}$. BRAZIL, Rondônia, Cacaulândia, 200 km SSE of Proto Velho, 140 masl, $10^{\circ} 18.0^{\prime} \mathrm{S}, 62^{\circ} 52.1^{\prime} \mathrm{W}$.

Previou record (Quate, 1996; Cordeiro et al., 2011): COSTA RICA, Limon, Puerto Viejo de Talamanca, sea level (type locality). BRAZIL, Amazonas, Manacapuru $74 \mathrm{~km}$ WSW of Manaus, $03^{\circ} 17^{\prime} 48.8^{\prime \prime} \mathrm{S}, 60^{\circ} 37^{\prime} 38.0^{\prime \prime} \mathrm{W}$.

\section{Psychoda trilobatula Cordeiro, Bravo \& Carvalho}

Psychoda trilobatula Cordeiro, Bravo \& Carvalho, 2011: 34-35, figs. 150-158. 
New record (determined by $D$. Cordeiro): BRAZIL, Rondônia, Cacaulândia, 200 km SSE of Porto Velho, 140 masl, $10^{\circ} 18.0^{\prime} \mathrm{S}, 62^{\circ} 52.1^{\prime} \mathrm{W}$.

Previous records (Cordeiro et al., 2011): BRASIL, Amazonas, Silves, Saracá (type locality); Amazonas, Manacapuru, $74 \mathrm{~km}$ WSW of Manaus, $03^{\circ} 17^{\prime} 48^{\prime \prime} \mathrm{S}$, 60 37'38'W; Amazonas, Itacoatiara-Itapiranga km 23, < 100 masl, $03^{\circ} 03.0^{\prime} \mathrm{S}, 58^{\circ} 43.5^{\prime} \mathrm{W}$; Pará, Santarém, Chac. N.S. Nazaré.

\section{Psychoda varablanca Quate}

Psychoda varablanca Quate, 1996: 77, figs. 31b-c.

New records (determined by D. Cordeiro): GUATEMALA, Izabal, Puerto S. Tomas Castillo, 10 masl. COSTA RICA, Limon, Puerto Viejo de Talaman, sea level, LS-401.000-600.000; Cartago, 30 km N of San Isidro, 2,640 masl, $09^{\circ} 32.7^{\prime} \mathrm{N}, 83^{\circ} 42.8^{\prime} \mathrm{W}$; Guanacaste, Monteverde, St. Elena, 1,500 masl, $10^{\circ} 19.1^{\prime} \mathrm{N}, 84^{\circ} 48.5^{\prime} \mathrm{W}$. VENEZUELA, Barinas, la Azulita, 1,400-1,900 masl, $08^{\circ} 42^{\prime} \mathrm{N}, 71^{\circ} 28^{\prime} \mathrm{W}$; Aragua, $17 \mathrm{~km}$ S of Choronf, 760 masl Aragua, 22 km S of Choronf, 1,000 masl; Aragua, 10 km N El Limon. PERU, Cuzco, Rio Madre de Dios, 28 km ESE of Boca Manu, 250 masl, $12^{\circ} 21^{\prime} \mathrm{S}, 70^{\circ} 42^{\prime} \mathrm{W}$. BRAZIL, Rondônia, Cacaulândia, 200 km SSE of Porto Velho, 140 masl, $10^{\circ} 18.0^{\prime} \mathrm{S}, 62^{\circ} 52.1^{\prime} \mathrm{W}$; Mato Grosso, Chapada dos Guimaraes, $35 \mathrm{~km}$ ENE of Cuiaba, $15^{\circ} 27^{\prime} 10^{\prime \prime} \mathrm{S}, 55^{\circ} 45^{\prime} 15^{\prime \prime} \mathrm{W}$.

Previous records (Quate, 1996): COSTA RICA, Heredia, Vara Blanca (type locality); Heredia, Puerto Viejo de Sarapaqui Est. Biol LaSelva, 50-100 masl, LN-268.000-535.600; San José, San Gerardo de Dota Alb. Savegre, 2,250 masl, $09^{\circ} 33^{\prime} 07^{\prime \prime} \mathrm{N}, 83^{\circ} 48^{\prime} 30^{\prime \prime} \mathrm{W}$.

\section{Psychoda zetoscota Quate, 1959}

Psychoda zetoscota Quate, 1959:214, figs. 1-2; Duckhouse, 1973: 13; Bravo et al., 2006: 7-8, figs. 9-18; Cordeiro et al., 2011: 18, figs. 34-35.

New record (determined by D. Cordeiro): BRAZIL, Rondônia, Cacaulândia, 200 km SSE of Porto Velho, 140 masl, $10^{\circ} 18.0^{\prime} \mathrm{S}, 62^{\circ} 52.1^{\prime} \mathrm{W}$.

Previous records (Quate, 1959; Bravo et al., 2006; Cordeiro et al., 2011): PANAMA, Zona do Canal (type locality). TRINIDAD, Tucker valley, US Naval Station. BRAZIL, Pará, Santarém, Ch. N.S. Nazaré; Bahia, Senhor do Bonfim, serra da Maravilha; Bahia, Cachoeira, faz. Villa Rial; Bahia, Ituberá, Pacangê; Paraná, Antonina, res. Sapitanduva.

\section{CONCLUSIONS}

This study of a well preserved and curated collection provided more than 70 new geographic records, which can be very helpful in future efforts of rapid assessments, as well as may contribute to studies on biogeography and conservation. The number of known species of Brazilian Psychodidae was also increased to 553, including one genus first time reported, also increasing to 46 the number of Psychodidae genera in Brazil.

\section{ACKNOWLEDGEMENTS}

I want to thank Dr. Brian Brown and Gian-Ann for the support on studying the specimens of LACM collection, and Dr. Lawrence Quate (in memorian) for the beautiful collection and many determined specimens. I'm also thankful for reviewers that contributed to this manuscript. This research received financial support of Programa Refauna of MCTIC/INPA to cover the expenses of the visit to LACM Museum, and the author had a post-doc fellowship provided by CAPES through PNPD Program.

\section{REFERENCES}

Aguiar, G.M. \& Vieira, V.R. 2018. Regional distribution and habitats of brazilian Phlebotomine species. In: Rangel, E.F. \& Shaw, J.J. (Eds.). Brazilian sand flies. Biology, taxonomy, medical importance and control. Cham, Switzerland, Springer. p. 251-298.

Bravo, F. \& Araújo, M.X. 2014. Psychodidae não Phlebotominae (Diptera) do Semiárido. In: Bravo, F. \& Calor, A. (Eds.). Atrópodes do Semiárido. Biodiversidade e Conservação. Feira de Santana, Printmídia. p. 279-284.

Bravo, F.; Cordeiro, D. \& Chagas, C. 2006. Two new species and new records of Psychoda Latreille (Diptera: Psychodidae: Psychodinae) from Brazil, with comments on supraspecific classification of the genus. Zootaxa, 1298: $1-15$

Collantes, F. \& Martinez-Ortega, E. 1999. Nuevas citas de espécies conocidas de Psychodidae (Diptera: Psychodidae) em Nicaragua. Revista Nicaraguense de Entomología, 48: 17-27.

Cordeiro, D. \& Bravo, F. 2008. A new species of viviparous moth fly (Diptera, Psychodidae, Psychodinae) from the Brazilian Amazon. Biota Neotropica, 8(2): 115-119. DOI

Cordeiro, D.; Bravo, F. \& Carvalho, C.J.B. 2011. Taxonomy of Brazilian Psychoda Latreille, 1796 (Diptera, Psychodidae) with the description of thirteen new species. Zootaxa, 3101: 1-37.

Curler, G.R. \& Moulton, J.K. 2008. A review of the Nearctic species of the genus Eurygarka Quate (Diptera: Psychodidae). Zootaxa, 1740: 28-36.

Duckhouse, D.A. 1973. Family Psychodidae. In: Papavero, N. (Ed.). A Catalogue of the Diptera of the Americas south of the United States. Museu de Zoologia, Universidade de São Paulo. 6A, p. 1-29.

Edwards, F.W. 1928. Nematocera. Insects of Samoa. London, British Museum (Natural History). pt. 6, 102p.

Quate, L.W. 1954. A revision of the Psychodidae of the Hawaiian Islands (Diptera). Proceedings of the Hawaiian Entomological Society, 15: 335-356.

Quate, L.W. 1955. A revision of the Psychodidae (Diptera) in America North of Mexico. University of California Publications in Entomology, 10: 103-273.

Quate, L.W. 1959. Taxonomy of Neotropical Psychodidae (Diptera) 1. Psychoda species of West Indies and Central America with a key to Trinidad species. The Pan-pacific Entomologist, 35: 213-221.

Quate, L.W. 1962. A taxonomic study of Borneo Psycchodinae (Diptera: Psychodidae). Pacific Insects, 4(1): 1-75. 
Quate, L.W. 1996. Preliminary taxonomy of Costa Rican Psychodidae (Diptera), exclusive of Phlebotominae. Revista de Biologia Tropical, Supplement 1, 44: 1-81.

Quate, L.W. 1999. Taxonomy of Neotropical Psychodidae (Diptera) 3. Psychodines of Barro Colorado Island and San Blas, Panama. Memoirs on Entomology International, 14: 409-441.

Quate, L.W. \& Brown, B.V. 2004. Revision of Neotropical Setomimini (Diptera: Psychodidae: Psychodinae). Contributions in Science, Natural History Museum of Los Angeles County, 500: 1-120.

Rafael, J.A. 2019. Diptera. In: Catálogo Taxonômico da fauna do Brasil. Available from: http://fauna.jbri.gov.br/fauna/faunadobrasil/252. Access in: 10/10/2019.

Rafael, J.A.; Melo, G.A.R.; Carvalho, C.J.B.; Casari, S.A. \& Constantino, R. 2012. Insetos do Brasil, diversidade e taxonomia. Riberão Preto, Holos.

Rangel, E.F. \& Lainson, R. 2009. Proven and putative vectors of American cutaneous leishmaniasis in Brazil: aspects of their biology and vectorial competence. Memórias do Instituto Oswaldo Cruz, 104: 937-954. D0I
Satchell, G.H. 1953. New and little known Samoan Psychodidae and a new species from Rarotonga. Proceedings of the Royal Entomological Society of London, 22: 181-188.

Shimabukuro, P.H.F.; Andrade, A.J.; Galati, E.A.B.; Cordeiro, D.P. \& Bravo, F. 2016. Psychodidae. In: Catálogo taxonômico da fauna do Brasil. Available at: http://fauna.jbri.gov.br/fauna/faunadobrasil/2258. Access in: 28/11/2018.

Shorthouse, D.P. 2010. SimpleMappr, an online tool to produce publicationquality point maps. Available at: https://www.simplemappr.net. Access in: 10/10/2019.

Tonnoir, A. 1922. Synopsis des espèces européennes du genre Psychoda (Diptères). Annales de la Societé Entomologique de Belgique, 62: 49-88.

Wagner, R. \& Ibáñez-Bernal, S. 2009. Psychodidae (sand flies, and moth flies or owl flies). In: Brown, B.V.; Borkent, A.; Cumming, J.M.; Wood, D.M.; Woodley, N.E. \& Zumbado, M. (Eds.). Manual of Central American Diptera. Ottawa, National Research Council of Canada. v. 1, p. 319-332. 